\title{
, \\ Fibrocartilaginous embolism of the cervical spinal cord and cerebellum in a cat
}

\author{
Kazuki OKADA $^{1)}$ and Yumiko KAGAWA ${ }^{1) *}$ \\ ${ }^{1)}$ North Lab, 8-35 Hondori 2-chome Kita, Shiroishi-ku, Sapporo, Hokkaido 003-0027, Japan
}

J. Vet. Med. Sci.

81(5): 747-749, 2019

doi: 10.1292/jvms.19-0037

Received: 17 January 2019

Accepted: 3 March 2019

Published online in J-STAGE:

12 March 2019
ABSTRACT. A 9-year-old castrated male cat presented with sudden onset of paralysis in four limbs. Magnetic resonance imaging revealed an intramedullary lesion at $\mathrm{C} 3$, and lesions in the vermis and right hemisphere of the cerebellum, which were hyperintense on T2-weighted imaging. The cat was euthanized, and postmortem examination was performed. Grossly, transverse sectioning of the spinal cord revealed areas of malacia within the gray matter extending from C2 to C3. Histologically, the ventral spinal artery, the spinal intramedullary arteries and the arterioles of arachnoid in the cerebellum contained fibrocartilaginous emboli confirmed by metachromatic staining with toluidine blue. To our knowledge, this is the first report of fibrocartilaginous embolism involving both the cerebellum and cervical spinal cord in a cat.

KEY WORDS: cat, cerebellum, cervical spinal cord, fibrocartilaginous embolism

Fibrocartilaginous embolism (FCE) has been well documented in dogs [4], sporadically in humans [1] and other species $[12,13]$. Clinical signs are often associated with a sudden onset of non-painful, non-progressive, and asymmetric paresis [6]. Magnetic resonance imaging (MRI) findings are helpful in the clinical diagnosis of FCE [1, 4], although histological examination is mandatory for a definitive diagnosis. FCE in cats has been reported previously [2, 9-11, 17], and these lesions were located exclusively in the spinal cord. Here we describe the first case of feline FCE involving both the cerebellum and spinal cord.

A 9-year-old, $4.5 \mathrm{~kg}$, castrated male domestic shorthaired cat exhibited sudden onset of paralysis in all four limbs in state of being recumbent. The cat had been kept exclusively indoors. Complete blood count and serum biochemical analyses were within normal limits, and thoracic and abdominal radiography was unremarkable. There were no external abrasions or any other evidence of trauma. A neurological examination revealed that the menace reflex was absent on the left side. Mental status was normal. Spinal reflexes of four limbs were normal. MRI revealed an intramedullary lesion at C3, and lesions in the vermis and right hemisphere of the cerebellum. These lesions were hyperintense on T2-weighted imaging (Fig. 1) and fluid attenuated inversion recovery imaging. They were hypointense on T1-weighted imaging, and did not exhibit contrast enhancement after intravenous administration of gadolinium. The MRI showed slight herniation of the cerebellar tonsils. Differential diagnoses included infarction, hemorrhage, or neoplasia. On the following day, the cat was euthanized, and postmortem examination was performed. Grossly, no significant lesions were noted in the brain and spinal cord up to C4. The brain and spinal cord samples were fixed in neutral buffered formalin. Transverse sectioning of the formalin-fixed spinal cord revealed malacic gray matter areas extending from C2 (Fig. 2) to C3. The border between cortex and medulla in the vermis of the cerebellum was unclear.

The samples were embedded in paraffin wax, and sections $(4-\mu \mathrm{m})$ were stained with hematoxylin and eosin (HE) and toluidine blue stains. Histologically, severe, regionally and bilateral necrosis of the ventral gray matter and right side dorsal gray matter was noted at $\mathrm{C} 2$ to $\mathrm{C} 3$. The ventral spinal artery and the intramedullary arteries contained homogeneous, faintly basophilic material (Fig. 3). The material was confirmed to be fibrocartilage by metachromatic staining with toluidine blue. The emboli composed of the material ranged from 15 to $30 \mu \mathrm{m}$ in diameter. In white matter tracts contiguous to areas of necrosis, marked vacuolation, numerous swollen axons, and gemistocytic astrocytes were observed. The emboli were also found in the arterioles of the arachnoid (Fig. 4) and capillary vessels of the cerebellar parenchyma, predominantly in the vermis and right hemisphere of the cerebellum. Numerous emboli were noted in the cerebellum, outnumbering those in the spinal cord; most of these were larger in diameter $(60-100 \mu \mathrm{m})$ than that of emboli in the spinal cord $(15-30 \mu \mathrm{m})$. The cerebellar cortex showed diffuse edema. Purkinje cells had hyper-eosinophilic or shrinkage cytoplasm and hyperchromatic nuclei. Neither emboli nor malacic foci were observed in the cerebrum, brain stem, or other areas of spinal cord.

In the previous reports of FCE in cats, the age range was between 4 and 12 years, and most cats were older than 7 years old $[6,9,10]$, similar to this case. The most commonly affected spinal cord segments were C6-T2. FCE has been reported more commonly in dogs, and a previous study of 393 dogs with FCE showed that 15 dogs (3.8\%) had neurological exam findings 


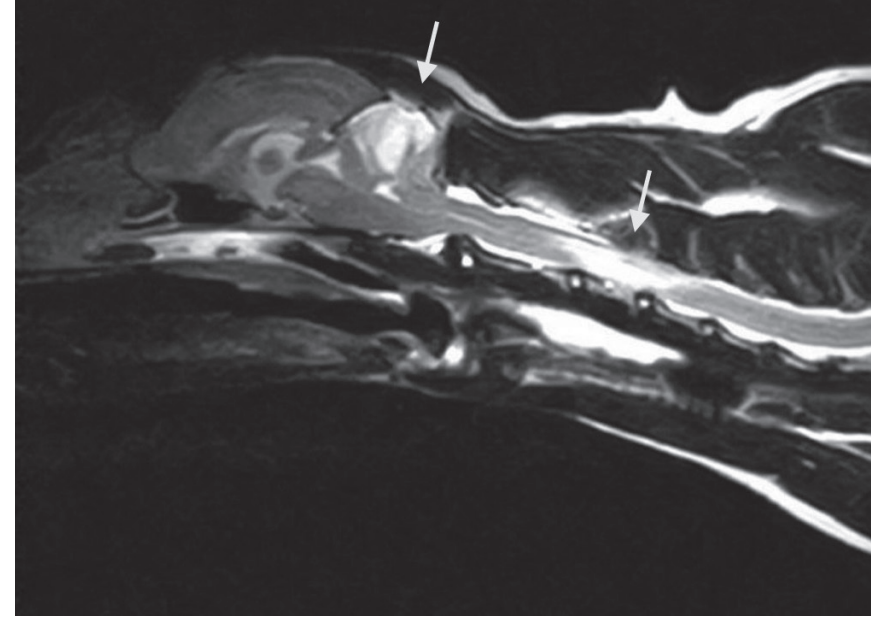

Fig. 1. A sagittal T2-weighted magnetic resonance image (repetition time $[\mathrm{TR}]=3,400$; echo time $[\mathrm{TE}]=120$ ) of the head and neck in a cat. There are hyperintense intraparenchymal lesions (arrows) at C3 and more extensively in the cerebellum.

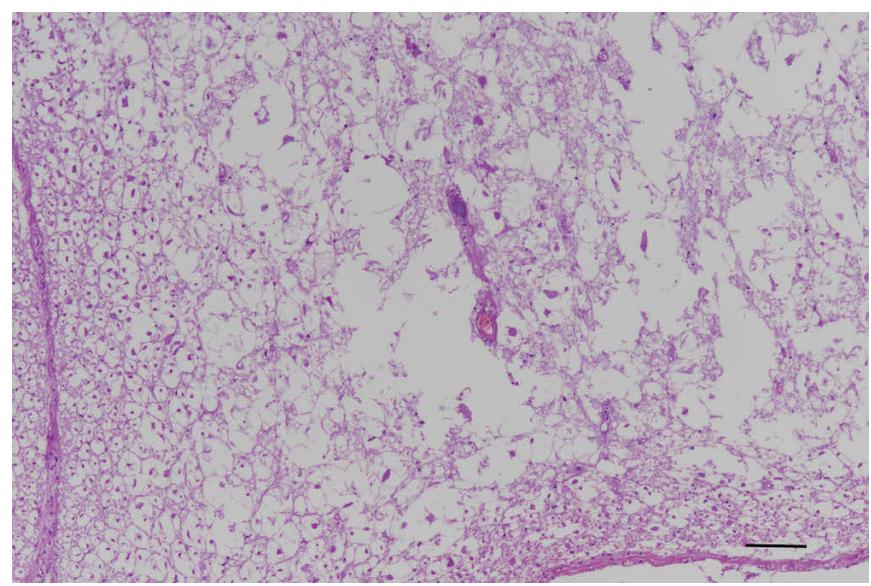

Fig. 3. Marked vacuolation, rarefaction and cavitation of the ventral white matter at the level of $\mathrm{C} 2$ in the cat. The intramedullary artery contained homogeneous, faintly basophilic material (embolus). H\&E. Bar $=100 \mu \mathrm{m}$.

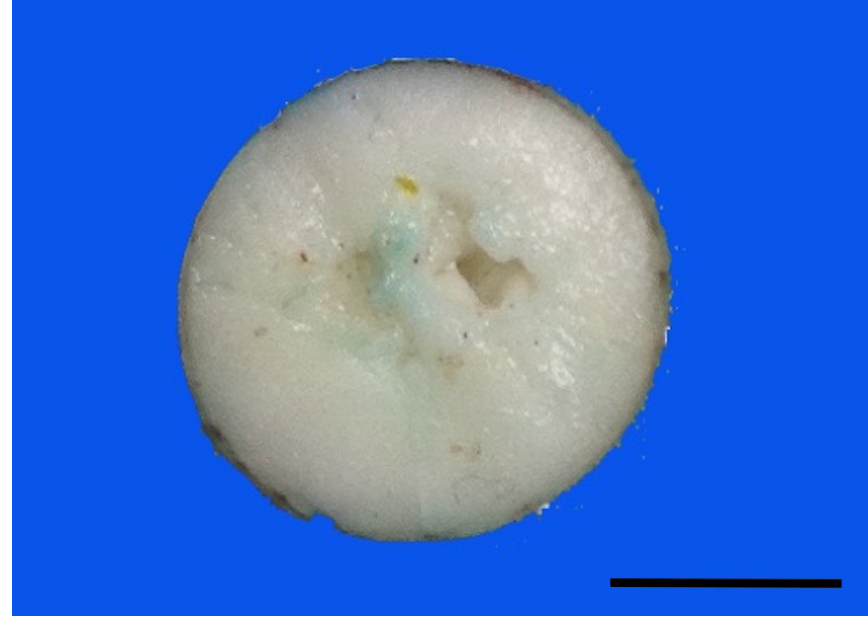

Fig. 2. Area of gray matter malacia at the level of spinal cord segment $\mathrm{C} 2$. Transverse section of formalin-fixed spinal cord in the cat. Bar $=5 \mathrm{~mm}$.

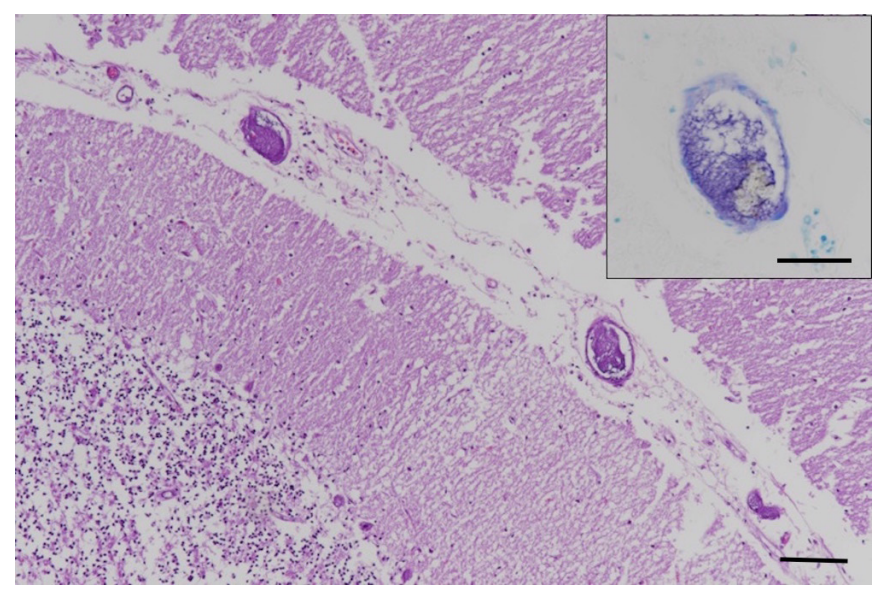

Fig. 4. Fibrocartilaginous emboli occupied the lumen of multiple arterioles in the arachnoid in the vermis of cerebellum in the cat. H\&E. Inset shows a high magnification of the embolus, which was metachromatic with toluidine blue staining. Toluidine blue stain. Bar $=100 \mu \mathrm{m}$; inset bar $=50 \mu \mathrm{m}$.

indicative of multifocal lesions in the spinal cord [4]. The most common multifocal lesions were the combination of T3-L3 and L4-S3 [4]. However, there is only one case report in a dog describing FCE involving the brainstem and mid-cervical spinal cord [3]. In dogs, the most common neuroanatomical location of FCE is at T3-L3 level [4], the distance between the brain and T3-L3 level is longer than the distance between the brain and cervical spinal cord where the emboli of this case were found in. The long distance between brain and affected areas of spinal cord (T3-L3) might explain why the emboli do not reach the brain in most cases of FCE. Although the prognosis of canine FCE is generally good [4], there was no improvement in a case involving the brain [3] as well as in the present feline case. FCE involving the brain may be associated with a poor prognosis. Among other species, one case of multifocal FCE involving cervical spinal cord, medulla oblongata and cerebellum has been reported in a neonatal sheep [8]. FCE in humans has been rarely reported, with $17 \%$ involving the medulla oblongata [1]. Only one case of FCE involving the middle cerebral artery has been reported in a human [14].

In the case reported herein, emboli were found in the ventral spinal artery, intramedullary arteries of the spinal cord, and arterioles of the arachnoid in the vermis and right hemisphere of the cerebellum. MRI findings suggested infarctions in C2 to C3 and in area of the cerebellum served by the right rostral cerebellar arteries. Arteries that supply blood to the cerebellum in cats are rostral and caudal cerebellar arteries [16]. Vertebral arteries of which ventral spinal arteries are branches reach the areas supplied by the caudal and rostral cerebellar vessels in cats [7]. The emboli may have traveled from the ventral spinal artery to the rostral 
cerebellar artery via the basilar artery.

In this case, histological change of the cerebellum (diffuse edema) was mild compared to that of the spinal cord (ischemic necrosis). The ventral spinal arteries supply specific areas of the spinal cord [15], while the fields supplied by the vertebral and carotid arteries overlap in the cerebellum [7]. There may also be differences in the time taken for emboli to become lodged in the brain and spinal cord, i.e. emboli arising initially in the spinal cord, followed by the cerebellum, may explain why the cerebellar ischaemia in this case was so subtle.

Fibrocartilaginous emboli histochemically have the appearance of the nucleus pulposus of the intervertebral discs, so they are presumed to originate from this source. Although several theories have been proposed in humans [1], and animals [5, 6], the routes which fibrocartilaginous materials enter blood vessels are unknown. In this case, the emboli in the cerebellum were noted to be greater in number and size, when compared to those in the cervical spinal cord. This suggests that a few emboli first could have entered only small blood vessels, e.g. central artery, later, many emboli could have entered directly large blood vessels, e.g. ventral spinal artery, and the emboli reached the cerebellum.

The unique distribution of the emboli in this case might contribute to elucidation of the mechanisms of entry of the fibrocartilaginous embolic material into the vasculature.

\section{REFERENCES}

1. AbdelRazek, M. A., Mowla, A., Farooq, S., Silvestri, N., Sawyer, R. and Wolfe, G. 2016. Fibrocartilaginous embolism: a comprehensive review of an under-studied cause of spinal cord infarction and proposed diagnostic criteria. J. Spinal Cord Med. 39: 146-154. [Medline] [CrossRef]

2. Abramson, C. J., Platt, S. R. and Stedman, N. L. 2002. Tetraparesis in a cat with fibrocartilaginous emboli. J. Am. Anim. Hosp. Assoc. 38: 153-156. [Medline] [CrossRef]

3. Axlund, T. W., Isaacs, A. M., Holland, M. and O'Brien, D. P. 2004. Fibrocartilaginous embolic encephalomyelopathy of the brainstem and midcervical spinal cord in a dog. J. Vet. Intern. Med. 18: 765-767. [Medline] [CrossRef]

4. Bartholomew, K. A., Stover, K. E., Olby, N. J. and Moore, S. A. 2016. Clinical characteristics of canine fibrocartilaginous embolic myelopathy (FCE): a systematic review of 393 cases (1973-2013). Vet. Rec. 179: 650. [Medline] [CrossRef]

5. Coradini, M., Johnstone, I., Filippich, L. J. and Armit, S. 2005. Suspected fibrocartilaginous embolism in a cat. Aust. Vet. J. 83: 550-551. [Medline] [CrossRef]

6. De Risio, L. and Platt, S. R. 2010. Fibrocartilaginous embolic myelopathy in small animals. Vet. Clin. North Am. Small Anim. Pract. 40: 859-869. [Medline] [CrossRef]

7. Holmes, R. L., Newman, P. P. and Wolstencroft, J. H. 1958. The distribution of carotid and vertebral blood in the brain of the cat. J. Physiol. 140: 236-246. [Medline] [CrossRef]

8. Jeffrey, M. and Wells, G. A. H. 1986. Multifocal ischaemic encephalomyelopathy associated with fibrocartilaginous emboli in the lamb. Neuropathol. Appl. Neurobiol. 12: 415-424. [Medline] [CrossRef]

9. MacKay, A. D., Rusbridge, C., Sparkes, A. H. and Platt, S. R. 2005. MRI characteristics of suspected acute spinal cord infarction in two cats, and a review of the literature. J. Feline Med. Surg. 7: 101-107. [Medline] [CrossRef]

10. Mikszewski, J. S., Van Winkle, T. J. and Troxel, M. T. 2006. Fibrocartilaginous embolic myelopathy in five cats. J. Am. Anim. Hosp. Assoc. 42: 226-233. [Medline] [CrossRef]

11. Scott, H. W. and O'Leary, M. T. 1996. Fibro-cartilaginous embolism in a cat. J. Small Anim. Pract. 37: 228-231. [Medline] [CrossRef]

12. Stedman, N. L., Brown, T. P. and Rowland, G. N. 1998. Intravascular cartilaginous emboli in the spinal cord of turkeys. Avian Dis. 42: $423-428$. [Medline] [CrossRef]

13. Tessaro, S. V., Doige, C. E. and Rhodes, C. S. 1983. Posterior paralysis due to fibrocartilaginous embolism in two weaner pigs. Can. J. Comp. Med. 47: 124-126. [Medline]

14. Toro-González, G., Navarro-Román, L., Román, G. C., Cantillo, J., Serrano, B., Herrera, M. and Vergara, I. 1993. Acute ischemic stroke from fibrocartilaginous embolism to the middle cerebral artery. Stroke 24: 738-740. [Medline] [CrossRef]

15. Vandevelde, M., Higgins, R. J. and Oevermann, A. 2012. Vascular disorders. p. 44. In: Veterinary Neuropathology: Essentials of Theory and Practice, 1st ed. (Vandevelde, M., Higgins, R. J. and Oevermann, A. eds.), Wiley Blackwell, West Sussex.

16. Whittaker, D. E., Drees, R. and Beltran, E. 2018. MRI and clinical characteristics of suspected cerebrovascular accident in nine cats. J. Feline Med. Surg. 20: 674-684. [Medline] [CrossRef]

17. Zaki, F. A., Prata, R. G. and Werner, L. L. 1976. Necrotizing myelopathy in a cat. J. Am. Vet. Med. Assoc. 169: 228-229. [Medline] 